\title{
iPreP is a three-dimensional nanofibrillar cellulose hydrogel platform for long-term ex vivo preservation of human islets
}

\author{
Yi-Ju Chen, ${ }^{1,2,3,4}$ Taiji Yamazoe, ${ }^{1}$ Karla F. Leavens, ${ }^{5,6}$ Fabian L. Cardenas-Diaz, ${ }^{5}$ Andrei Georgescu, ${ }^{7}$ \\ Dongeun Huh, ${ }^{7}$ Paul Gadue, ${ }^{5}$ and Ben Z. Stanger ${ }^{1,2,3}$ \\ ${ }^{1}$ Castroenterology Division, Department of Medicine, 'Department of Cell and Developmental Biology, and ${ }^{3}$ Abramson \\ Family Cancer Research Institute, Perelman School of Medicine, University of Pennsylvania, Philadelphia, Pennsylvania, \\ USA. ${ }^{4}$ Cenetic Resource Science, The Jackson Laboratory, Bar Harbor, Maine, USA. ${ }^{5}$ Center for Cellular and Molecular \\ Therapeutics, Department of Pathology and Laboratory Medicine, 'Division of Endocrinology, Department of Pediatrics, \\ Children's Hospital of Philadelphia, Philadelphia, Pennsylvania, USA. 'Department of Bioengineering, School of \\ Engineering and Applied Sciences, University of Pennsylvania, Philadelphia, Pennsylvania, USA.
}

Islet transplantation is an effective therapy for achieving and maintaining normoglycemia in patients with type 1 diabetes mellitus. However, the supply of transplantable human islets is limited. Upon removal from the pancreas, islets rapidly disintegrate and lose function, resulting in a short interval for studies of islet biology and pretransplantation assessment. Here, we developed a biomimetic platform that can sustain human islet physiology for a prolonged period ex vivo. Our approach involved the creation of a multichannel perifusion system to monitor dynamic insulin secretion and intracellular calcium flux simultaneously, enabling the systematic evaluation of glucose-stimulated insulin secretion under multiple conditions. Using this tool, we developed a nanofibrillar cellulose hydrogel-based islet-preserving platform (iPreP) that can preserve islet viability, morphology, and function for nearly 12 weeks ex vivo, and with the ability to ameliorate glucose levels upon transplantation into diabetic hosts. Our platform has potential applications in the prolonged maintenance of human islets, providing an expanded time window for pretransplantation assessment and islet studies.

Conflict of interest: The authors have declared that no conflict of interest exists.

Copyright: (c) 2019, American Society for Clinical Investigation.

Submitted: August 31, 2018

Accepted: September 20, 2019

Published: November 1, 2019 .

Reference information: /CI Insight. 2019;4(21):e124644.

https://doi.org/10.1172/jci.

insight.124644.

\section{Introduction}

Diabetes mellitus (DM) has become a global public health threat — affecting more than 30 million people in the United States (9.4\% of the population) and 415 million people worldwide - leading to significant health care expenditures (1-3). DM can be classified into type 1 (T1DM) and type 2 (T2DM), both of which are characterized by hyperglycemia resulting from the destruction and/or dysfunction of pancreatic islets. Normally, $\beta$ cells within pancreatic islets function to precisely regulate blood glucose levels by secreting insulin in response to a meal. The chronic imbalance of blood glucose levels in patients with DM leads to systemic organ damage, including cardiovascular disease, blindness (retinopathy), kidney failure (nephropathy), and nerve damage (neuropathy)

Islet transplantation, in which human islets isolated from organ donors are infused into the portal vein of the liver, is an effective treatment for severe diabetes (4-7). However, a paucity of suitable donors, technical challenges associated with tissue processing, and islet loss during pretransplantation culture have hindered more widespread use of this treatment. Hence, methods to more effectively maintain the function of human islets for extended periods of time ex vivo may help to ease these supply issues, either by increasing the availability of donor-derived human islets or by providing an optimal environment for the propagation of functional insulin-producing cells generated from various stem cells (8-11).

Islets occupy only approximately $1 \%-2 \%$ of the total pancreas mass, but they are well vascularized and receive $15 \%$ of the blood flow $(12,13)$. In addition, each islet is surrounded by an extracellular matrix (ECM), constituting a structural framework that provides mechanical and chemical signals that sustain essential features of islet physiology (e.g., morphology, differentiation, survival, and insulin secretion) (14). Standard islet isolation methods employ enzymatic digestion steps that destroy most ECM proteins $(14,15)$. 
A

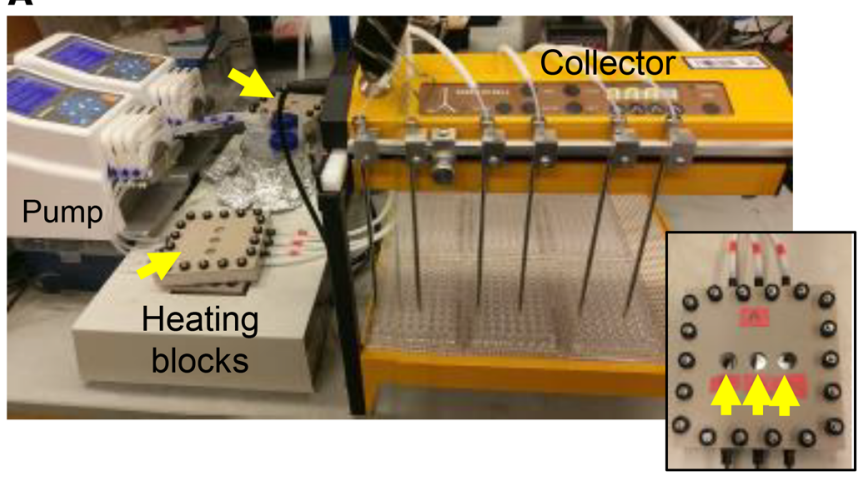

C

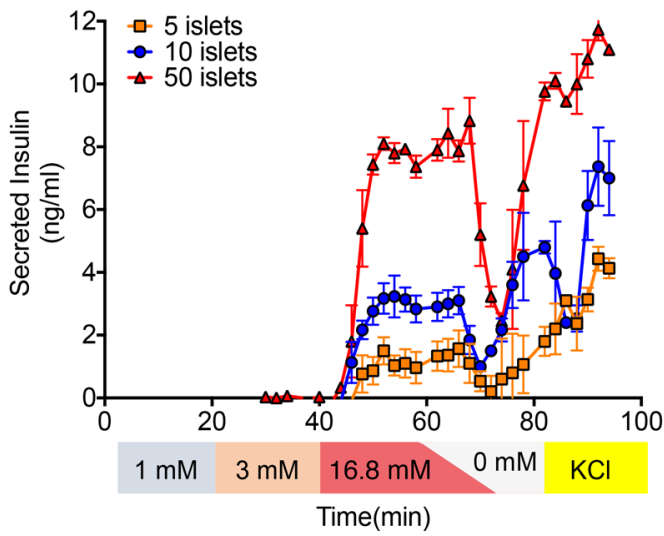

B

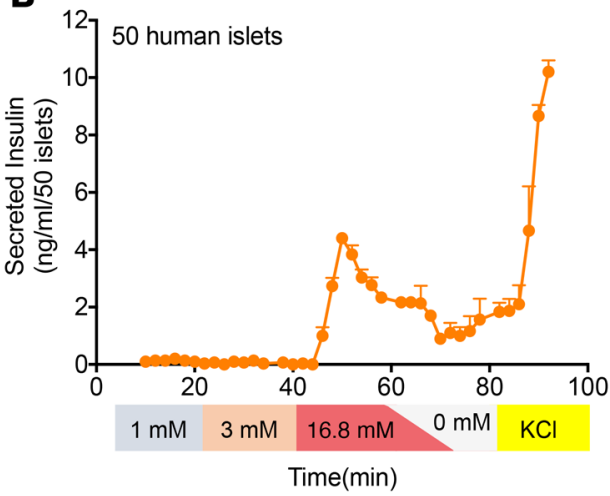

D

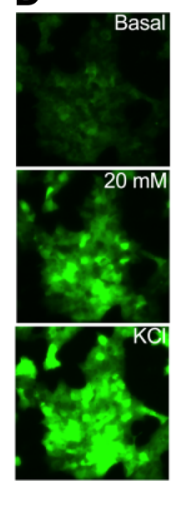

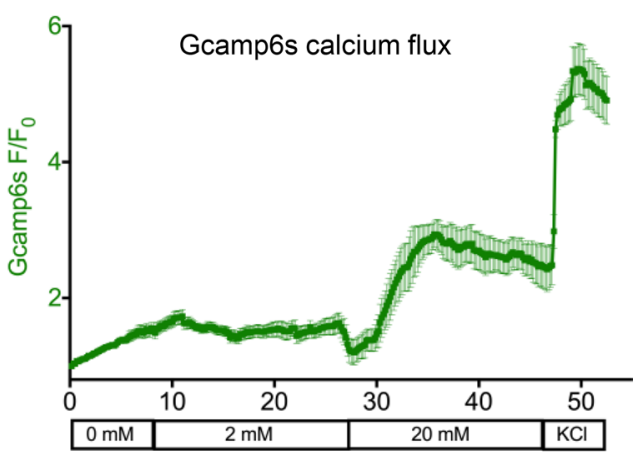

Figure 1. Custom multichannel perifusion system with real-time imaging capacity. (A) The multichannel perifusion system, consisting of two peristaltic pumps, two 3-channel perifusion chambers (yellow arrows), heating blocks, tubing, and a 6-channel fraction collector. Inset: Enhanced view of an individual 3-channel perifusion chamber (yellow arrows indicate optical chambers). (B) Measurement of GSIS using the custom multichannel perifusion system. Fifty human islets per channel, each from the same donor, were perfused in triplicate with $1 \mathrm{mM}, 3 \mathrm{mM}$, and $16.8 \mathrm{mM}$ glucose, followed by a short period of KRBB buffer and $30 \mathrm{mM} \mathrm{KCl}$ (see Methods for details). Fractions were collected every minute, and insulin concentrations were measured by HTRF insulin assay. Experiments were repeated 10 times with islets isolated from donors shown in Table 1. Results from a single representative experiment are shown. Each data point represents the mean \pm SEM of insulin measurement ( $n=3$ replicates per time point). (C) Sensitivity of the multichannel perifusion system. Five, 10, or 50 human islets from the same donor were loaded into each well of a perifusion chamber, followed by perifusion as described in B. Each curve represents a single experiment ( $n=3$ replicates per time point). Each data point represents mean \pm SEM of insulin measurement. (D) Real-time imaging analysis of calcium influx in EndoC- $\beta \mathrm{H} 1$ cells in response to glucose stimulation. EndoC- $\beta \mathrm{H} 1$ cells were engineered to express a calcium-sensitive green fluorescence reporter (GCaMP6s) under the control of the rat insulin promoter. Cells were placed into the perifusion chamber, mounted on an inverted epifluorescence microscope, and subjected to perifusion using the indicated concentrations of glucose and $\mathrm{KCl}$ (see Methods). Experiments were performed in triplicate and results plotted as mean fluorescence intensity of the whole field versus time (sampling every 10 seconds).

This disruption of the ECM proteins can promote islet fragmentation and cell clumping, significantly reducing islet viability and function. Furthermore, standard culture methods are associated with a high rate of islet attrition independent of the isolation process, even in brief culture periods (16). To address these problems, we used high-throughput functional assays to evaluate various in vitro culture parameters, including matrix and medium composition, to identify conditions that prevent the disintegration of human islets and preserve their viability and function for extended periods of time ex vivo.

\section{Results}

A multichannel perifusion system for assessing islet physiology. To facilitate the identification of an appropriate ex vivo environment for human islets, we established a high-throughput system to rapidly assess islet function (Figure 1A). The system utilizes a pump to establish continuous flow through a custom-designed 3-channel perifusion chamber that holds islet samples. The inflow tracts are designed to allow an easy exchange of the incoming buffer solution, with minimal air bubble generation and dead volumes, permitting islets to be exposed to varying concentrations of glucose or other compounds with short lag times. Effluent from the 
Table 1. Characteristics of donors and human islets used in this study

\begin{tabular}{|c|c|c|c|c|c|c|c|}
\hline Donor ID & Sex & Age (yr) & BMI & Ethnicity & HbA1c (\%) & BG (mg/dL) & $\begin{array}{l}\text { Culture time prior to shipping } \\
\text { from the center }\end{array}$ \\
\hline 1 & M & 37 & 27.6 & White & 5.6 & 174.2 & 19 hours \\
\hline 2 & M & 15 & 24.6 & Hispanic/Latino & 5.1 & 122.2 & 2 days 19 hours \\
\hline 3 & M & 60 & 26.6 & White & 5.9 & 146 & 3 days 20 hours \\
\hline 4 & M & 49 & 26.3 & White & 5.1 & 166.8 & 1 day 20 hours \\
\hline 5 & $\mathrm{~F}$ & 32 & 27.9 & Hispanic/Latino & 5.6 & 179.8 & 9.1 hours \\
\hline 8 & M & 55 & 30.1 & White & 5.6 & 152 & 2 days \\
\hline 9 & M & 46 & 34.4 & White & 5.6 & 162.4 & 1 day 8 hours \\
\hline 10 & M & 38 & 33 & White & 4.6 & 140.2 & 3 days \\
\hline
\end{tabular}

perifusion chamber is fed into a 6-channel fraction collector, allowing samples to be saved for later measurement of insulin or other secreted molecules.

To assess the performance of the device, we loaded 50 human islets into the perifusion chamber and dynamically perifused them with low- $(1 \mathrm{mM}$ or $3 \mathrm{mM})$ or high-glucose $(16.8 \mathrm{mM})$ solutions. The serial fractions of effluent were measured for human insulin by ELISA (characteristics of the human islet donors are presented in Table 1). At the end of all perifusion experiments, islets were exposed to $\mathrm{KCl}$, which acts as a membrane-depolarizing agent to stimulate $\mathrm{Ca}^{2+}$ influx, causing the exocytosis of remaining insulin granules from the cell. Using the multichannel setup, we observed robust insulin release under high-glucose conditions and reduced levels of insulin release under low-glucose conditions (Figure 1B). Furthermore, we were able to detect glucose-stimulated insulin secretion (GSIS) with as few as 5 human islets (Figure 1C).

Under physiological conditions, GSIS is known to be coupled to the metabolic state of the $\beta$ cell and dependent on an influx of $\mathrm{Ca}^{2+}(17)$. To enable real-time imaging of $\mathrm{Ca}^{2+}$ flux in $\beta$ cells, we designed the perifusion chamber to have an ultrathin Gorilla glass window, allowing dynamic visualization of intracellular imaging profiles during perifusion. We then introduced the GCaMP6s calcium sensor protein, which undergoes a $\mathrm{Ca}^{2+}$-dependent conformation change resulting in increased GFP fluorescence (18), into the immortalized human $\beta$ cell line EndoC- $\beta$ H1 (19), and performed simultaneous perifusion and imaging. As shown in Figure 1D (and Supplemental Video 1; supplemental material available online with this article; https://doi.org/10.1172/jci.insight.124644DS1), we observed low-level oscillations of GFP fluorescence at $0 \mathrm{mM}$ (buffer) and $2 \mathrm{mM}$ glucose, indicating that EndoC- $\beta \mathrm{H} 1$ cells have minimal levels of intracellular $\mathrm{Ca}^{2+}$ flux under low-glucose conditions. By contrast, an increase in GFP fluorescence was observed in the presence of $20 \mathrm{mM}$ glucose or $\mathrm{KCl}$. Finally, we demonstrated that this system permits simultaneous measurements of glucose-dependent insulin release and real-time measurements of calcium flux (Supplemental Figure 1). These results indicate that our perifusion chamber can be used to correlate changes in intracellular $\mathrm{Ca}^{2+}$ flux with cellular physiology.

Dynamic insulin secretion profiles and morphology of human islets in different media and $2 D$ culture platform. Standard islet culture methods are associated with a loss of islet architecture, function, and survival over a period of days (20). We thus used our multichannel perifusion system to evaluate the effects of various combinations of different culture media, culture vessels, and 3D matrices on islet physiology. Because CMRL 1066-based media - specifically CMRL, PIM, and CIT — have been reported to support human islet culture (20-24), we began with a direct comparison of the ability of these different media formulations to support islet function (Figure 2, A and B). Human islets were loaded into 96-well culture plates with CMRL, PIM, or CIT; placed in a tissue culture incubator set to standard conditions $\left(37^{\circ} \mathrm{C}, 5 \% \mathrm{CO}_{2}\right)$; and functionally assessed on day 1, 14, or 21 of culture. As shown in Figure 2A, islets cultured in CMRL medium for 14 days (black curve) exhibited a significant decrease in function and elevated insulin release under low-glucose conditions compared with fresh isolated islets from the same donor tested 1 day after receipt (gray curve). By contrast, islets cultured in PIM (green) or CIT (red) exhibited robust insulin release in high-glucose conditions, comparable to that of fresh (1 day) islets, although basal levels of insulin release in low-glucose 

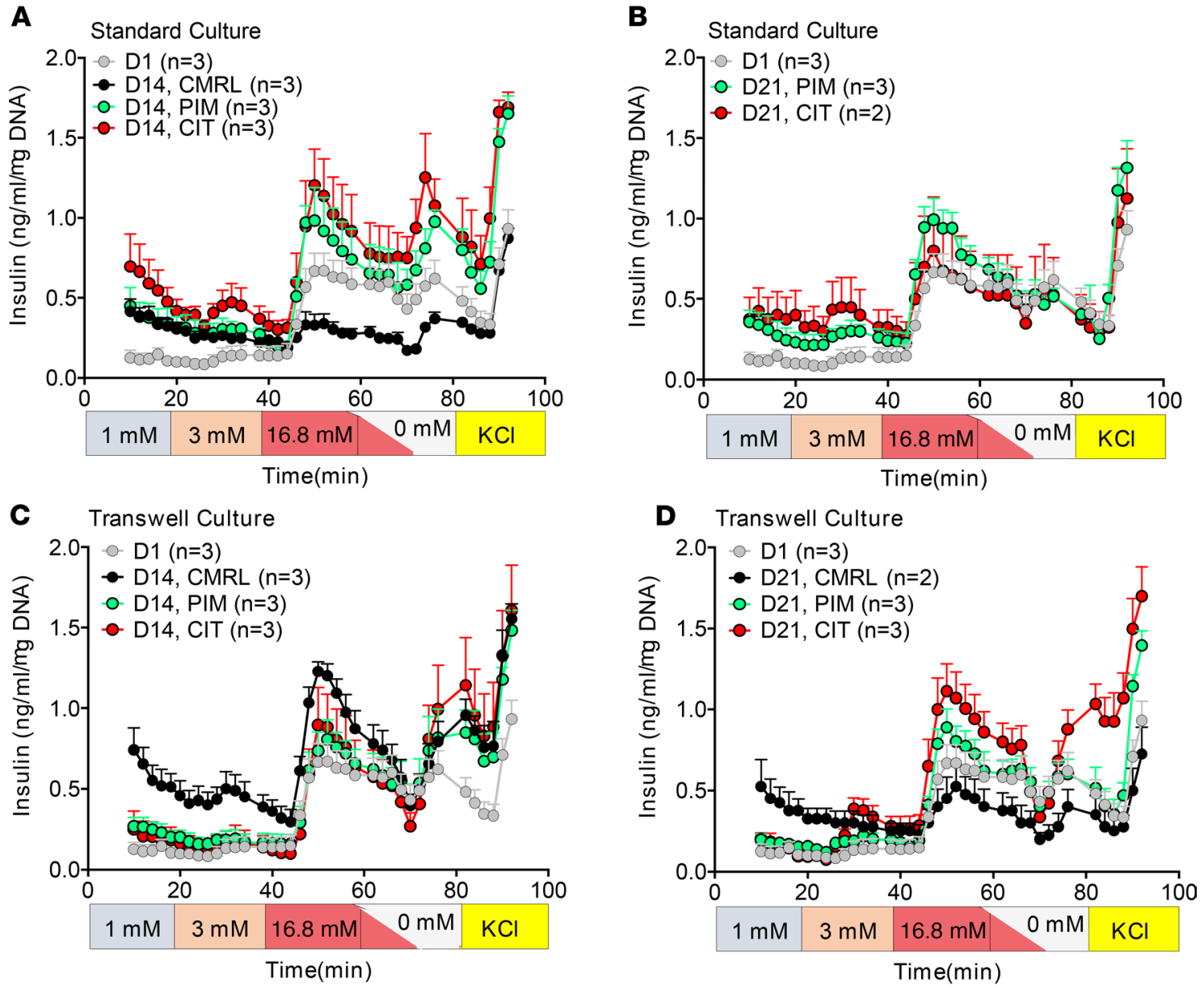

$\mathbf{E}$

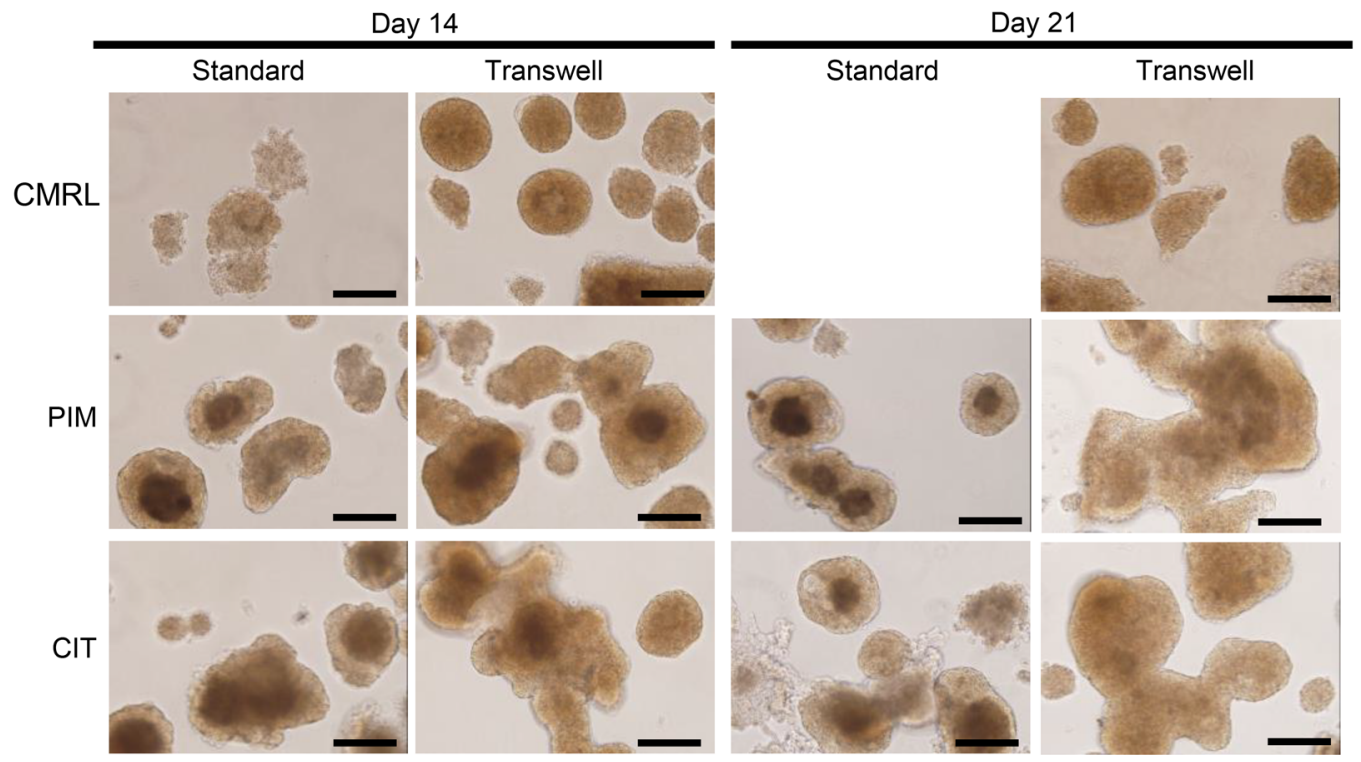

Figure 2. Effect of CMRL 1066-based media and Transwell filters on the function of human islets during long-term culture. (A-D) Fifty handpicked human islets from the same donor per replicate were cultured in a standard platform (96-well plate) (A and B) or Transwell filter (C and D) with different CMRL 1066-based media (CMRL, PIM, or CIT). Islet function in each replicate was assessed on day 1, 14, or 21 by perifusion. Results were obtained from 3 independent experiments, and each experiment was conducted in duplicate or triplicate with islets isolated from the same donor. The number of donors used in each condition $(n=2$ or $n=3)$ is indicated. All data were pooled for each condition shown and plotted as mean \pm SEM. All insulin values were normalized to DNA content of each sample. (E) Representative morphological assessment of long-term-cultured human islets. Human islets cultured in PIM or CIT medium remained viable but exhibited an aggregated morphology, with a darkening core reflecting central necrosis. Islets cultured in CMRL exhibited a loss of viability after 14-day culture. Scale bars: $100 \mu \mathrm{m}$. 
conditions were still higher than normal (Figure 2A). These improved GSIS patterns remained intact after 21 days of PIM or CIT culture (Figure 2B), whereas GSIS could not be evaluated after 21 days of CMRL culture due to islet disintegration and/or death. Islets cultured with PIM or CIT also remained competent to release insulin following membrane depolarization with $30 \mathrm{mM} \mathrm{KCl}$ (Figure 2B).

In vivo, islets are perfused by a dense vascular network that ensures a sufficient supply of oxygen and nutrients, facilitating robust sampling and modulation of glucose concentration (25). In addition, islets have a specialized microenvironment that provides physical support and other biologically relevant signals (14). Standard ex vivo culture conditions fail to reproduce either of these features, as islets are subjected to relative hypoxia (as a consequence of vessel loss) and exposed to significant physical stresses due to a rigid substratum (stiffness of $\sim 10^{6} \mathrm{kPa}$ in a tissue culture dish versus $\sim 1-2 \mathrm{kPa}$ in the native pancreas [refs. 26, 27]). Consequently, we sought conditions that could both improve oxygenation and better reproduce the normal physical microenvironment of the islet. To this end, we cultured islets on semipermeable Transwell filters using the medium conditions in Figure 2, A and B, to provide a less-rigid surface. An added advantage of Transwell culture is that the islets are overlaid with a minimal amount of medium, resulting in a greater potential for gas exchange.

Human islets cultured on Transwell filters exhibited robust GSIS in all medium conditions including CMRL - when assayed after 14 days (Figure 2C) or 21 days (Figure 2D) in culture. While islets cultured on Transwell filters in CMRL continued to exhibit high levels of basal insulin secretion, those cultured in PIM or CIT exhibited tight control of insulin release in low-glucose conditions, resulting in GSIS profiles that were comparable to those of freshly isolated islets (Figure 2, C and D). Nevertheless, evaluation of islet morphology revealed a tendency for islets to aggregate and develop a central necrotic core regardless of the culture conditions (Figure 2E). Taken together, a multiparametric comparison of different medium preparations and culture environments indicate that $2 \mathrm{D}$ islet culture with permeable support (Transwell filter) in the presence of CIT or PIM medium maintains islet function for 2-3 weeks ex vivo. However, these conditions do not preserve islet morphology or prevent the darkening of the central core of the islet.

$3 D$ nanofibrillar cellulose hydrogels maintain long-term islet function and morphology. Our Transwell experiments suggested that physical forces may play a role in the maintenance of islet function ex vivo. To better recapitulate the stiffness of the native pancreatic microenvironment and provide added $3 \mathrm{D}$ support for the islets, we tested a number of extracellular matrices for the ability to preserve islet morphology, settling on hydrogels made from nanofibrillar cellulose (NFC; GrowDex) for further experiments. NFC hydrogel is a wood-derived material with a nanofibrillar network composed of aligned $\beta$-D- $(1 \rightarrow 4)$ glucopyranose polysaccharide chains (27). NFC fiber networks have a constant stiffness that is easily tunable without a need for further cross-linking, and their viscoelastic properties are similar to those of the native ECM $(28,29)$. Importantly, the NFC hydrogel can be enzymatically degraded by cellulase - which has little or no activity in animal tissues - to retrieve cultured cells or organoids.

Given these favorable properties, we incorporated NFC hydrogels into the Transwell culture to create a $3 \mathrm{D}$ biomimetic human islet culture platform, which we refer to as an islet-preserving platform, or $i$ PreP (Figure 3A). Human islets were encapsulated with $0.75 \%$ NFC hydrogel $(\mathrm{w} / \mathrm{v})$, loaded onto Transwell filters, and incubated with CIT medium, followed by viability and morphology assessment after 21 days of culture. Islet morphology and viability were well maintained in this 3D NFC hydrogel platform (Figure 3A). Next, we investigated the function of human islets after long-term 3D NFC culture by handpicking 50 human islets and culturing them in the iPreP platform for 2-6 weeks. Islets cultured in the cellulose hydrogel for 14 or 21 days exhibited insulin secretion profiles similar to those of fresh isolated islets ( 1 day), including low levels of insulin secretion in low-glucose conditions and robust insulin release in response to $16.8 \mathrm{mM}$ glucose or $\mathrm{KCl}$ (Figure 3B). Next, we directly compared the performance of the 3D NFC hydrogel platform with that of $2 \mathrm{D}$ Transwell culture by incubating human islets for 42 days in the two conditions and examining GSIS function. As shown in Figure $3 \mathrm{C}$, islets in the 3D NFC hydrogel platform continued to maintain function throughout the culture period comparable to their freshly isolated counterparts (1 day). By contrast, islets cultured directly on Transwell filters for 42 days exhibited high levels of basal insulin secretion in low glucose (Figure 3C). Total DNA count did not change during the culture period, indicating that cell numbers were maintained, although total insulin content exhibited a 2 -fold reduction in both Transwell and hydrogel conditions (Supplemental Figure 2). 
A

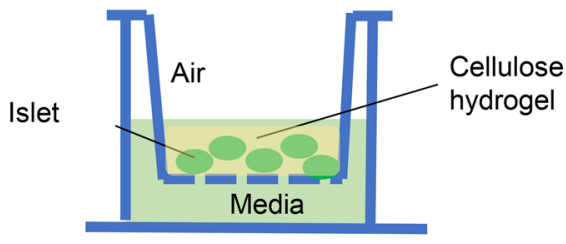

Day 21, Hydrogel

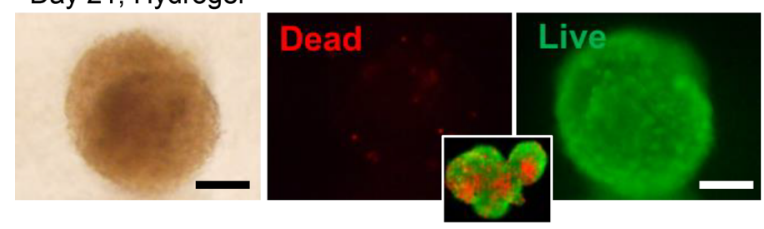

C

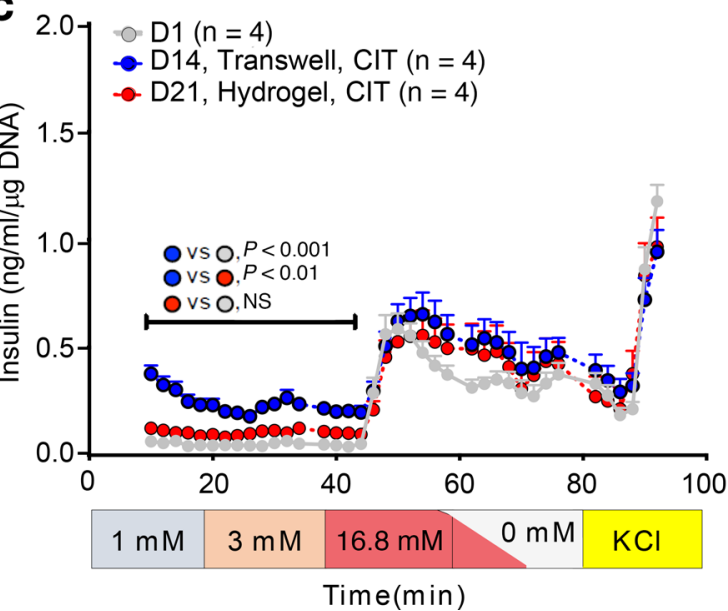

B

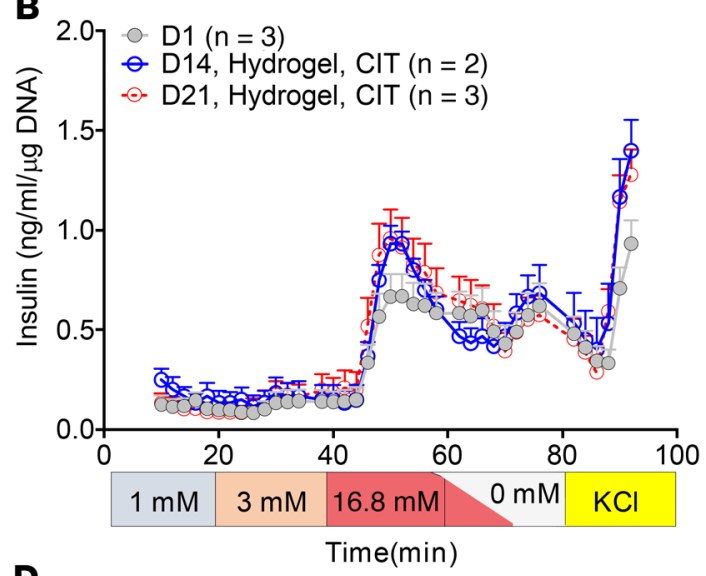

D

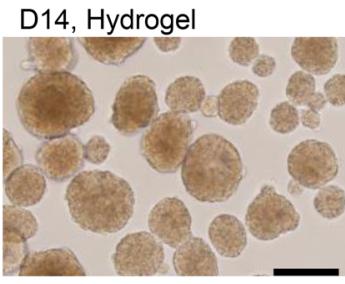

D21, Hydrogel

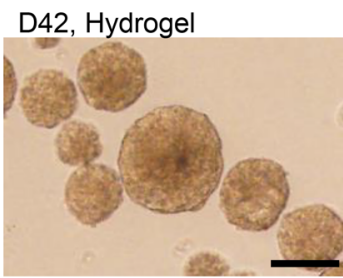

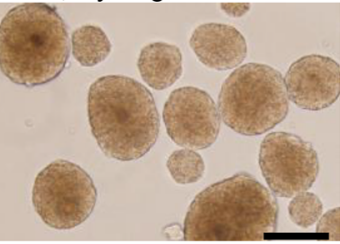

D83, Hydrogel

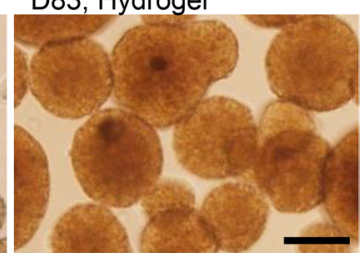

Figure 3. A 3D NFC hydrogel platform for maintaining human islet function and morphology in a long-term. (A) Viability assessment of human islets encapsulated in a 3D NFC hydrogel platform. Top: Schematic of the 3D NFC hydrogel platform. Bottom: Representative images of an islet after 21 days cultured in NFC hydrogel platform, followed by LIVE/DEAD viability staining and imaged for morphology (bright-field), viable cells (green fluorescence), and apoptotic cells (red fluorescence). Inset: Representative image of an islet cultured for 7 days in standard (CIT) medium, followed by LIVE/DEAD staining. Scale bar: $100 \mu \mathrm{m}$. (B) Human islets cultured for 14 or 21 days in NFC hydrogel with CIT media were functionally similar to their freshly isolated (day 1) counterparts. The experiments were conducted using islets from the same donors as in Figure 2 . Results were obtained from 3 independent experiments. Each experiment was performed in duplicate or triplicate with islets isolated from the same donor. The number of donors used in each condition $(n=2$ or $n=3)$ is indicated. Values are shown as mean \pm SEM. Insulin values were normalized to DNA content of each sample. (C) A comparison of human islets cultured for 42 days in Transwell or with 3D NFC hydrogels. Human islets cultured in Transwell for 42 days exhibited significantly higher levels of basal insulin secretion in response to low glucose ( $1 \mathrm{mM}$ and $3 \mathrm{mM})$ compared with fresh islets (day 1) or hydrogel-cultured islets. Experiments were conducted 4 times using samples from 4 different cadaveric donors. Each experiment was performed in duplicate or triplicate with islets isolated from the same donor. Values were shown as mean \pm SEM. Insulin values were normalized to DNA content of each sample. Multiple-comparisons $t$ test was performed, followed by Holm-Šidák correction. (D) Representative images of human islets following long-term NFC hydrogel culture. Cultured human islets were retrieved on day 14, 21, 42, or 83 by treatment with cellulase and imaged for morphology (bright-field). Scale bars: $100 \mu \mathrm{m}$.

Furthermore, the morphology of human islets retrieved from NFC hydrogels exhibited a well-rounded spheroid shape with clear membrane borders and minimal central darkening, which was only observed in large islets $(>150-200 \mu \mathrm{m})$, for culture periods as long as 83 days (Figure 3D). These results demonstrate that islet cultures using the iPreP platform preserve human islet viability, function, and morphology for more than a month ex vivo.

Functional assessment of long-term cultured human islets in vivo. To further explore the potency of these long-term cultured human islets, we performed islet transplantation following iPreP culture. Two hundred handpicked human islets were cultured in either iPreP or Transwell filter conditions for 31 days at $37^{\circ} \mathrm{C}$ in CIT medium, followed by transplantation under the kidney capsule of STZ-induced diabetic NOD/SCID mice. Transplantation of islets cultured using iPreP, but not those cultured directly on Transwell filters, resulted in a significant lowering of blood glucose, although blood glucose levels began to climb in both transplanted groups at later time points (Figure 4A). 

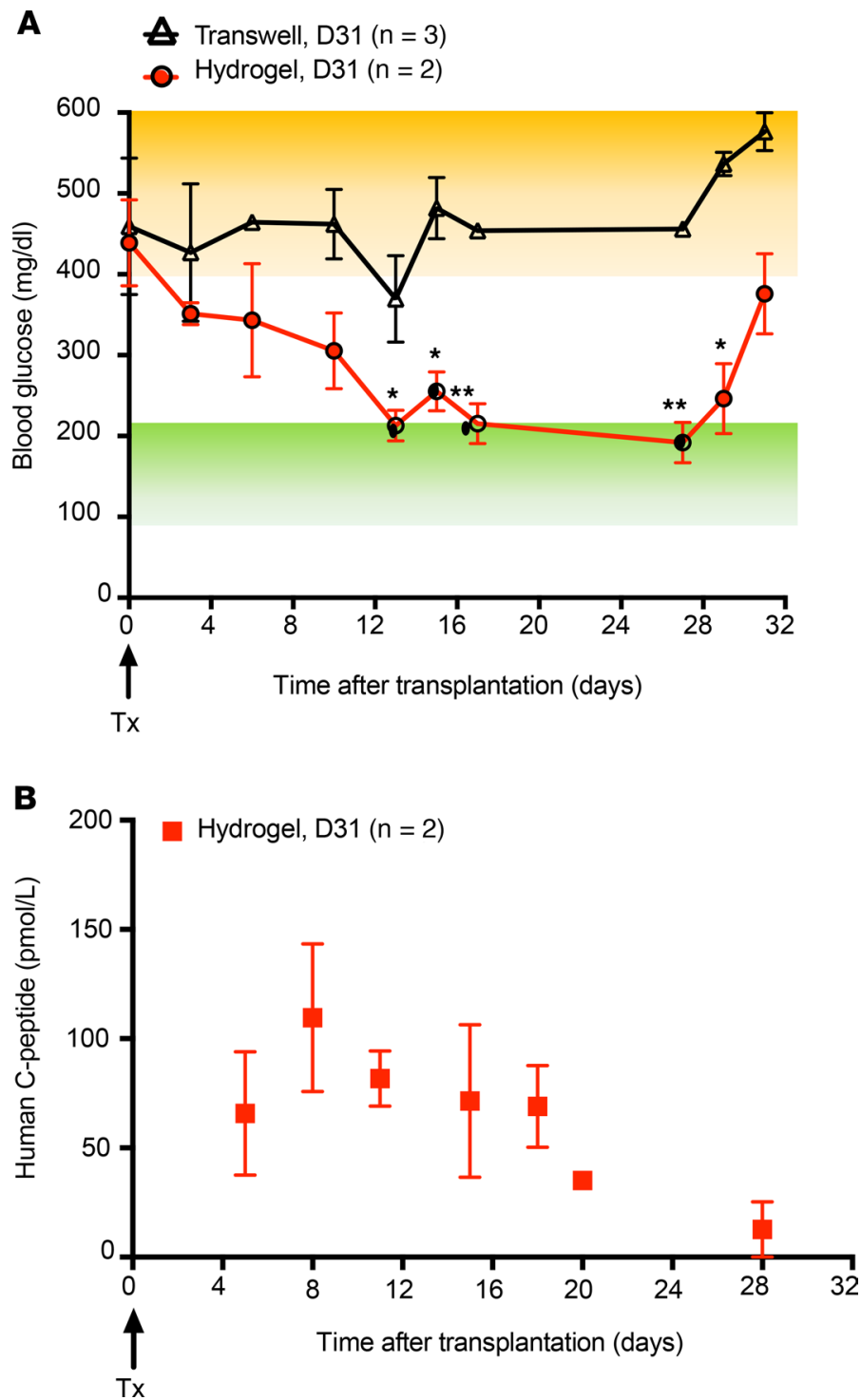

Figure 4. In vivo functional assessment of human islets following long-term 3D NFC hydrogel culture. (A) Blood glucose levels in diabetic mice after transplantation of human islets cultured ex vivo for 31 days. Transwell- or hydrogel-cultured human islets from the same donor ( 200 islets per mouse, counted on day 0 ) were retrieved on day 31 and transplanted under the kidney capsule of STZ-induced diabetic NOD/SCID mice. Three mice were transplanted with Transwell cultured islets, and 2 mice were transplanted with hydrogel-cultured islets. Values are shown as mean \pm SEM. Yellow indicates hyperglycemia; green, normoglycemia. Two-tailed $t$ test; ${ }^{*} P<0.05,{ }^{*} P<$ 0.01. (B) Human C-peptide levels in diabetic mice after transplantation. Hydrogel-cultured human islets from the same donor (400 islets per mouse, counted on day 0 ) were retrieved on day 31 and transplanted into 2 STZ-induced diabetic NOD/SCID mice. Blood samples were collected from mouse tail vein at the indicated time points after transplantation, and human C-peptide levels were measured from serum. Data are shown as mean \pm SEM.

To confirm that the transplanted islets were functional, we performed another transplantation experiment using different donor islets and measured human C-peptide levels in mouse serum on specific days after transplantation (Figure 4B). On day 5 after transplantation, human C-peptide levels reached $65.8 \pm 28.25 \mathrm{pmol} / \mathrm{L}$, peaking on day 8 after transplantation at $109.6 \pm 33.80 \mathrm{pmol} / \mathrm{L}$ and then ranging from $69 \mathrm{pmol} / \mathrm{L}$ to $82 \mathrm{pmol} / \mathrm{L}$ from day 11 to day 18 after transplantation. Human C-peptide levels dropped off subsequently, with concentrations of $35.09 \pm 0.17 \mathrm{pmol} / \mathrm{L}$ and $12.63 \pm 12.63 \mathrm{pmol} / \mathrm{L}$ on day 20 and day 28 after transplantation, respectively. Taken together, these results indicate that this NFC hydrogel platform can sustain human islet function during long-term ex vivo culture. 


\section{Discussion}

In this study, we sought to determine how differences in medium composition, culture environment, and matrix might impact islet physiology after long-term culture. It has previously been shown that CMRL 1066 medium supplemented with L-glutamine and FBS is superior for culturing human islets compared with RPMI 1640 medium, which was widely used in the islet studies and more suitable for culturing mouse islets (20). This CMRL formulation is able to support human islet GSIS function for up to 7 days ex vivo. However, xenogeneic bovine serum cannot be used in human islet culture for clinical transplantation. Thus, human serum or human albumin has been used to replace FBS in CMRL-based medium (PIM or CIT) for short periods (24-72 hours) of islet pretransplantation culture $(7,21-23,29)$.

Using a multichannel perifusion device to test multiple variables in parallel, we found that each condition tested - medium, culture environment, and matrix - had an impact on islet survival and function. Medium composition, the first parameter we tested, had a major effect on islet performance over a 2- to 3-week culture period. After 14 days, islets cultured in CIT or PIM had a marked improvement in function compared with islets cultured in standard CMRL, which exhibited a flattened appearance and lacked the ability to release insulin in response to glucose stimulation. This difference was even more apparent after 21 days, as CMRL-cultured islets had largely disintegrated and formed cell sheets in the petri dishes (data not shown), whereas CIT- or PIM-cultured islets remained viable and exhibited robust insulin release in response to high-glucose conditions. Further improvements in function and morphology were observed when islets were cultured on Transwell semipermeable membranes, a condition that we hypothesize leads to improved islet nutrient exchange and oxygenation as a result of their closer proximity to the gaseous phase.

Despite the improved function observed when islets were cultured on Transwell filters with either CIT or PIM, these conditions did not prevent the islet fusion and central necrosis that accompanies prolonged islet culture. Wood-derived NFC hydrogels, which provide mechanical support and limit islet motion, rendered islets both morphologically and functionally intact for more than 5 weeks. While large islets are more susceptible to hypoxia-induced central necrosis during culture, leading to significant loss of islet mass and function and unfavorable clinical outcomes following islet transplantation $(30,31)$, most iPreP-cultured islets did not develop central necrosis, and only a few large islets $(>150 \mu \mathrm{m})$ had central darkening of the core. Importantly, the functional preservation conferred by iPreP was measurable in vivo. Usually, $500-1000$ islet equivalents (IEQ) of freshly isolated islets are needed to reverse diabetes in a mouse model $(32,33)$. In our hands, 200-400 long-term iPreP-cultured islets could significantly ameliorate blood glucose levels in STZ-treated mice, even after ex vivo culture periods greater than 4 weeks. These results strongly indicate that our biomimetic iPreP platform - consisting of optimized medium, Transwell filters, and NFC hydrogels with proper stiffness - allows human islets to maintain a functional profile comparable to that of freshly isolated islets.

There are several potential applications from our findings. First, the identification of optimized islet maintenance conditions may augment efforts to generate functionally mature insulin-producing cells from pluripotent cells or other sources $(8-11,34)$. While several protocols have been developed to promote the directed differentiation of embryonic stem cells (ESCs) into insulin-positive $\beta$-like cells $(35,36)$, the products of those differentiation protocols function poorly in culture and lack robust GSIS profiles. Thus, conditions that preserve the function of bona fide islets - particularly with respect to low basal insulin secretion, a hallmark of $\beta$ cell maturity - may enhance the performance of islets generated from alternative cellular sources.

Second, the development of a platform for long-term islet culture expands the time window for conducting studies of human islet physiology. Despite previous efforts to extend the ex vivo life span of human islets $(22,37-39)$, they remain notoriously difficult to maintain in culture. Currently, most experiments must be performed within days following the harvesting of islets from an organ donor. Because human islet availability is unpredictable, research teams must be able to rapidly mobilize to receive donor islets and conduct experiments before islet function declines. The culture conditions described here may thus give researchers greater flexibility. Our platform could also be exploited for drug development, where the time constraints imposed by a limited islet supply hinders the ability to perform high-throughput drug screens. Such screens could facilitate the discovery of compounds to improve $\beta$ cell replication, maintenance, and/or secretory function.

Finally, the iPreP culture platform presents an opportunity to create a bank of human islets for basic investigation or clinical use. For certain organs, such as kidney and bone marrow, advanced donor identification allows for better planning and donor-recipient matching prior to transplantation, an option not available for islet transplantation given the poor viability and function of islets cultured for more than a few days. As our in vitro GSIS and in vivo transplantation experiments show, human islets can retain full function when 
cultured under suitable conditions. Because the NFC hydrogel used in this study is derived from wood and is xenogenic-free, the culture platform described here - if appropriately scaled — could in principle be adapted to create an "islets-on-demand" infrastructure to support research and clinical transplantation needs.

\section{Methods}

Experimental setup for multichannel GSIS perifusion assay. Two 3-channel perifusion digital pumps (Ismatec, Cole Parmer) were used to supply the solutions to the two 3-channel perifusion chambers. Both input solutions and perifusion chambers were prewarmed to $37^{\circ} \mathrm{C}$, and kept in a warm water bath and heating blocks as shown in Figure 1A. A flow rate was set for both pumps to achieve collection of fractions with a total volume of $300 \mu \mathrm{L}$ every minute in a well of 96 -well plate. Fifty handpicked human islets from a cadaveric donor were loaded into each well of the perifusion chamber. Human islets were first perfused for 3 minutes with warm Krebs-Ringer bicarbonate buffer (KRBB, $115 \mathrm{mmol} / \mathrm{L} \mathrm{NaCl}$, $24 \mathrm{mmol} / \mathrm{L} \mathrm{NaHCO}_{3}, 5 \mathrm{mmol} / \mathrm{L} \mathrm{KCl}, 1 \mathrm{mmol} / \mathrm{L} \mathrm{MgCl}_{2}, 2.5 \mathrm{mmol} / \mathrm{L} \mathrm{CaCl}_{2}, 10 \mathrm{mM} \mathrm{HEPES}, 0.25 \%$ $\mathrm{BSA}, \mathrm{pH}$ 7.4) without glucose followed by another 3 minutes with $1 \mathrm{mM}$ glucose-KRBB buffer prior to a fraction collection step. To collect the fractions, islets were then perfused in a sequence with $1 \mathrm{mM}$ glucose for 16 minutes, $3 \mathrm{mM}$ glucose for 20 minutes, $16.8 \mathrm{mM}$ glucose for 24 minutes, KRBB buffer for 18 minutes, and $30 \mathrm{mM} \mathrm{KCl-KRBB}$ buffer for 12 minutes. Fractions were collected every minute in 96-well plates. Secreted insulin was measured from the collected fractions by HTRF insulin assay using human insulin as a standard (Cisbio). The secreted insulin values were consistent across replicate wells. The results represent experiments done in duplicate or triplicate with a single-donor islet preparation. Data are shown as mean \pm SEM of each point. We reproducibly detected dynamic insulin secretion in human islets isolated from 10 healthy nondiabetic organ donors (see Human islet culture and Table 1).

Calcium imaging. An immortalized human $\beta$ cell line (EndoC- $\beta \mathrm{H} 1$ ) was transduced with RIP-GCaMP6s lentivirus to generate a stable cell line, EndoC- $\beta$ H1-RIP-GCaMP6, which expressed a green fluorescence calcium sensor driven by the rat insulin promoter. To image calcium flux under different stimuli, the EndoC- $\beta$ H1-RIP-GCaMP6 cells were seed onto a lumox 96-well plate (SARSTEDT) and used for experiments at approximately $80 \%$ confluence. The film with cells was removed from the lumox plate, loaded into the perifusion chamber, and mounted on an inverted epifluorescence microscope (Leica 8900 series), followed by perifusion with a continuous flow of KRBB buffer $(0 \mathrm{mM}, 2 \mathrm{mM}, 20 \mathrm{mM}$ glucose, and $25 \mathrm{mM}$ $\mathrm{KCl}$ ). Quantification of calcium flux was performed by capturing images every 10 seconds. Results were plotted as mean fluorescence intensity of the whole field versus time.

For simultaneous measurement of insulin secretion and real-time calcium imaging, islets were briefly digested with $0.025 \%$ trypsin for 2 minutes, then incubated overnight with lentivirus expressing the genetically encoded calcium indicator protein GCamp6s under the control of the rat insulin promoter. The medium was changed the next morning, and 90 islets were perfused and imaged 48 hours later. Insulin was assayed from perfusate using Cisbio HTRF Insulin Assay. Fluorescence images were taken every minute using an inverted Leica microscope. The fluorescence intensity change $\left(\mathrm{F} / \mathrm{F}_{0}\right)$ of 3 separate regions within the field over background was determined using ImageJ software (NIH).

Human islet culture. Human islets were provided by the Integrated Islet Distribution Program (IIDP) and Penn Center for Islet Transplantation. The experiments were performed in accordance with policies and procedures of the IIDP and the University of Pennsylvania. On the day of arrival, human islets were handpicked into the tubes (50 islets per tube) and assigned to different groups to investigate the effect of medium, Transwell filter, and 3D NFC hydrogel culture. CMRL medium was CMRL 1066 (Corning) supplemented with $10 \%$ heat-inactivated FBS, $1 \times$ GlutaMAX (Life Technologies), $100 \mathrm{U} / \mathrm{mL}$ penicillin, and $100 \mu \mathrm{g} / \mathrm{mL}$ streptomycin. PIM(R) (Prodo Laboratories Inc.) medium was supplemented with $5 \%$ human $\mathrm{AB}$ serum and provided by the Penn Center for Islet Transplantation. CIT medium was prepared from CMRL 1066, CIT Modification (Corning) supplemented with $0.5 \%$ human albumin, $10 \mathrm{U} / \mathrm{mL}$ heparin, and $0.1 \mu \mathrm{g} / \mathrm{mL}$ IGF-1 (21). For standard culture, 50 islets were distributed into the well of a 96-well plate (Corning) and cultured in CMRL 1066, PIM, or CIT medium. Transwell Permeable Inserts, $6.5 \mathrm{~mm}$ with 8 - $\mu \mathrm{m}$ pores (Corning), were used in both Transwell culture and 3D NFC hydrogel culture. To set up the Transwell culture, 50 islets were suspended and loaded into the insert with CMRL, PIM, or CIT medium. For 3D NFC hydrogel culture, 50 human islets were mixed with $0.75 \%$ (w/v) NFC hydrogel (GrowDex; UPM Biomedicals) and then dispensed into the insert of Transwell. CIT medium was added to the top of hydrogel and the lower compartment. The culture media were replenished every 3-4 days. 
In vivo assessment of long-term-cultured human islets. A single injection of streptozotocin (STZ [MilliporeSigma]; $200 \mu \mathrm{g} / \mathrm{g}$ body weight) was administrated intraperitoneally to induce diabetes in NOD/SCID mice (The Jackson Laboratory). Diabetes was confirmed by fed blood glucose level $>400 \mathrm{mg} / \mathrm{dL}$ for 2 consecutive measurement. Mice that were not hyperglycemic 6 days after STZ administration were excluded from further study. Human islets from a single donor were handpicked on day 0 and cultured in Transwell or NFC hydrogel for 31 or 83 days. Islets were retrieved and transplanted under the kidney capsule of diabetic NOD/SCID mice (200 or 400 islets counted on day 0 per condition per mouse). After transplantation, fed blood glucose levels were measured in the late morning $(10 \mathrm{am}-12: 00 \mathrm{pm})$ of the indicated day with a OneTouch Ultra glucometer (LifeScan). Nephrectomy was done on day 31 after transplantation to remove graft-bearing kidneys in diabetic recipient mice. Serum human C-peptide levels were measured in mouse tail blood collected at the indicated time point by using an ultrasensitive C-peptide ELISA kit (Mercodia).

Statistics. Data are presented as mean \pm SEM or SD of individual groups as described in each figure legend. Differences between groups were analyzed by 2 -tailed Student's $t$ test. A $P$ value less than 0.05 was considered statistically significant.

Study approval. All animal experiments were approved by the Penn Institutional Animal Care and Use Committee (protocol 804572).

\section{Author contributions}

YJC, TY, KFL, FLCD, AG, DH, PG, and BZS were involved in designing the study, performing experiments, and analyzing data. BZS supervised the research and provided advice. TY performed islet kidney transplantation. KFL and FLCD performed calcium influx imaging and data analysis. YJC and BZS wrote the manuscript with input from all authors.

\section{Acknowledgments}

We thank A. Naji and C. Liu at Penn Center for islet Transplantation for providing human islets and clinical islet culture media; C. Li at CHOP for sharing the insulin HTRF microplate reader; M. Carman at Penn Research Instrument shop for making the multi-channel perifusion chamber; R.J. Norgard and S. Yuan for comments on the manuscript. This work was supported by the funding from NIH/NIDDK (UC4-DK104196, UG3-DK122644), Integrated Islet Distribution Program (IIDP), and Abramson Family Cancer Research Institute.

Address correspondence to: Ben Z. Stanger, 421 Curie Blvd, 512 BRB II/III, Philadelphia, Pennsylvania 19104, USA Phone: 215.746.5559; Email: bstanger@upenn.edu. Or to: Yi-Ju Chen, 600 Main Street, 01D 1115, Bar Harbor, Maine 04609, USA. Phone: 207.288.6217; Email: yijuchen3@gmail.com.

1. Zimmet P, Alberti KG, Magliano DJ, Bennett PH. Diabetes mellitus statistics on prevalence and mortality: facts and fallacies. Nat Rev Endocrinol. 2016;12(10):616-622.

2. CDC. National Diabetes Statistics Report 2017. http://www.cdc.gov/features/diabetes-statistic-report/index.html. Updated August 8, 2017. Accessed September 3, 2019.

3. International Diabetes Federation. Diabetes Atlas 2017.8th ed. http://diabetesatlas.org/IDF_Diabetes_Atlas_8e_interactive_ EN. Accessed September 3, 2019.

4. Brennan DC, et al. Long-term follow-up of the Edmonton protocol of islet transplantation in the United States. Am J Transplant. 2016;16(2):509-517.

5. Shapiro AM. Islet transplantation in type 1 diabetes: ongoing challenges, refined procedures, and long-term outcome. Rev Diabet Stud. 2012;9(4):385-406.

6. Shapiro AM, et al. Islet transplantation in seven patients with type 1 diabetes mellitus using a glucocorticoid-free immunosuppressive regimen. $N$ Engl J Med. 2000;343(4):230-238.

7. Shapiro AM, Pokrywczynska M, Ricordi C. Clinical pancreatic islet transplantation. Nat Rev Endocrinol. 2017;13(5):268-277.

8. Chen YJ, et al. De novo formation of insulin-producing "neo- $\beta$ cell islets" from intestinal crypts. Cell Rep. 2014;6(6):1046-1058

9. Cheng X, et al. Self-renewing endodermal progenitor lines generated from human pluripotent stem cells. Cell Stem Cell. 2012;10(4):371-384

10. Kroon E, et al. Pancreatic endoderm derived from human embryonic stem cells generates glucose-responsive insulin-secreting cells in vivo. Nat Biotechnol. 2008;26(4):443-452.

11. Talchai C, Xuan S, Kitamura T, DePinho RA, Accili D. Generation of functional insulin-producing cells in the gut by Foxo1 ablation. Nat Genet. 2012;44(4):406-412.

12. Lifson N, Kramlinger KG, Mayrand RR, Lender EJ. Blood flow to the rabbit pancreas with special reference to the islets of Langerhans. Gastroenterology. 1980;79(3):466-473.

13. Lifson N, Lassa CV, Dixit PK. Relation between blood flow and morphology in islet organ of rat pancreas. Am J Physiol. 
1985;249(1 pt 1):E43-E48.

14. Stendahl JC, Kaufman DB, Stupp SI. Extracellular matrix in pancreatic islets: relevance to scaffold design and transplantation. Cell Transplant. 2009;18(1):1-12.

15. Irving-Rodgers HF, Choong FJ, Hummitzsch K, Parish CR, Rodgers RJ, Simeonovic CJ. Pancreatic islet basement membrane loss and remodeling after mouse islet isolation and transplantation: impact for allograft rejection. Cell Transplant. 2014;23(1):59-72.

16. Daoud J, Rosenberg L, Tabrizian M. Pancreatic islet culture and preservation strategies: advances, challenges, and future outlook. Cell Transplant. 2010;19(12):1523-1535.

17. Satin LS. Localized calcium influx in pancreatic beta-cells: its significance for Ca2+-dependent insulin secretion from the islets of Langerhans. Endocrine. 2000;13(3):251-262.

18. Chen TW, et al. Ultrasensitive fluorescent proteins for imaging neuronal activity. Nature. 2013;499(7458):295-300.

19. Ravassard P, et al. A genetically engineered human pancreatic $\beta$ cell line exhibiting glucose-inducible insulin secretion. $J$ Clin Invest. 2011;121(9):3589-3597.

20. Holmes MA, Clayton HA, Chadwick DR, Bell PR, London NJ, James RF. Functional studies of rat, porcine, and human pancreatic islets cultured in ten commercially available media. Transplantation. 1995;60(8):854-860.

21. NIH CIT Consortium Chemistry Manufacturing Controls Monitoring Committee, NIH CIT Consortium. Purified human pancreatic islets, CIT culture media: a standard operating procedure of the NIH Clinical Islet Transplantation Consortium. CellR4 Repair Replace Regen Reprogram. 2014;2(3):e981.

22. Fraga DW, Sabek O, Hathaway DK, Gaber AO. A comparison of media supplement methods for the extended culture of human islet tissue. Transplantation. 1998;65(8):1060-1066.

23. Kerr-Conte J, et al. Upgrading pretransplant human islet culture technology requires human serum combined with media renewal. Transplantation. 2010;89(9):1154-1160.

24. Nacher M, et al. Human serum versus human serum albumin supplementation in human islet pretransplantation culture: in vitro and in vivo assessment. Cell Transplant. 2016;25(2):343-352.

25. Ballian N, Brunicardi FC. Islet vasculature as a regulator of endocrine pancreas function. World J Surg. 2007;31(4):705-714

26. Shi Y, Glaser KJ, Venkatesh SK, Ben-Abraham EI, Ehman RL. Feasibility of using 3D MR elastography to determine pancreatic stiffness in healthy volunteers. J Magn Reson Imaging. 2015;41(2):369-375.

27. Venkatesh SK, Ehman RL. Magnetic resonance elastography of abdomen. Abdom Imaging. 2015;40(4):745-759.

28. Bhattacharya M, et al. Nanofibrillar cellulose hydrogel promotes three-dimensional liver cell culture. J Control Release. 2012;164(3):291-298

29. Miron-Mendoza M, Seemann J, Grinnell F. The differential regulation of cell motile activity through matrix stiffness and porosity in three dimensional collagen matrices. Biomaterials. 2010;31(25):6425-6435.

30. Lehmann R, et al. Superiority of small islets in human islet transplantation. Diabetes. 2007;56(3):594-603.

31. Zorzi D, et al. Impact of islet size on pancreatic islet transplantation and potential interventions to improve outcome. Cell Transplant. 2015;24(1):11-23

32. Loganathan G, et al. Factors affecting transplant outcomes in diabetic nude mice receiving human, porcine, and nonhuman primate islets: analysis of 335 transplantations. Transplantation. 2013;95(12):1439-1447.

33. Suzuki K, et al. Function and survival of macroencapsulated syngeneic islets transplanted into streptozocin-diabetic mice. Transplantation. 1998;66(1):21-28.

34. Ariyachet $\mathrm{C}$, et al. Reprogrammed stomach tissue as a renewable source of functional $\beta$ cells for blood glucose regulation. Cell Stem Cell. 2016;18(3):410-421.

35. Pagliuca FW, et al. Generation of functional human pancreatic $\beta$ cells in vitro. Cell. 2014;159(2):428-439.

36. Rezania A, et al. Reversal of diabetes with insulin-producing cells derived in vitro from human pluripotent stem cells. Nat Biotechnol. 2014;32(11):1121-1133.

37. Fraga DW, Gaber AO, Kotb M. Long-term culture and maintenance of human islets of Langerhans in memphis serum-free media. Methods Mol Med. 2005;107:303-311.

38. Gaber AO, Fraga D. Advances in long-term islet culture: the Memphis experience. Cell Biochem Biophys. 2004;40(3 suppl):49-54.

39. Gaber AO, Fraga D, Kotb M, Lo A, Sabek O, Latif K. Human islet graft function in NOD-SCID mice predicts clinical response in islet transplant recipients. Transplant Proc. 2004;36(4):1108-1110. 